\title{
conditions de formation des surfaces de glissement conditions of slip surfaces formation
}

\author{
P. HABIB \\ Laboratoire de Mécanique des Solides * \\ (E.P., E.N.S.M. Paris, E.N.P.C., \\ Associé au C.N.R.S.)
}

\section{Résumé}

La formation des surfaces de glissement est étudiée pour des matériaux radoucissants comme les sables denses dilatants, les matériaux thermosensibles ou les argiles raides dans le schéma expérimental de la déformation plane de cisaillement. Le radoucissement est une instabilité plastique. Le mécanisme de déformation qui minimise la puissance dissipée correspond à la localisation de la déformation lorsque la résistance au cisaillement est une fonction décroissante du glissement.

\footnotetext{
Abstract

Slip surfaces formation is studied for work-softening materials as dense sands with dilatancy, thermosensitive medias or hard clays with the experimental set-up of plane shear strain. Softening is a plastic instability. The strain mechanism which give the smallest dissipated power correspond to the strain localisation when the shear stress is a decreasing fonction of the shear strain.
} 


\section{INTRODUCTION}

La formation des surfaces de glissement dans les sables denses, les matériaux thermosensibles et les argiles raides est étudiée ici par la recherche de mécanisme de déformation plastique qui dissipe la plus petite énergie, c'est-à-dire du mécanisme le plus défavorable.

Lorsqu'un sable dense est déformé d'une façon homogène, il est bien connu que son volume augmente dans des proportions non négligeables, qui peuvent atteindre et dépasser $15 \%$. D'autre part, plus la densité d'un sable est faible, moins sa résistance au cisaillement est grande. Enfin, comme la dilatation qui accompagne la déformation ne peut se poursuivre indéfiniment, il est assez intuitif que cette situation risque de devenir instable, c'est-à-dire que la déformation peut devenir hétérogène avec formation de surfaces de glissement.

Pour les matériaux thermosensibles l'évolution du phénomène est curieusement assez comparable à celle des sables denses. Les matériaux thermosensibles dont il est fait mention ici sont ceux dont la résistance diminue avec la température. C'est généralement le cas de la plupart des corps et plus particulièrement au voisinage du point de fusion. Au cours de la déformation plastique l'énergie dissipée se transforme en chaleur, la température augmente d'une façon aussi homogène que la déformation et, par conséquent, la résistance diminue dans tout le corps. Mais il suffit d'une hétérogénéité de la matière pour que la situation devienne instable : une faiblesse locale entraîne une plus grande déformation locale, d'où une plus grande élévation locale de température accompagnée d'une diminution de la résistance. Il semble que ce soit J. POMEY (1964) qui ait le premier décelé dans cette situation un risque d'instabilité et de localisation de la déformation sur une surface de glissement. Cet effet est particulièrement net pour les métaux à haute température : il faut, d'une part, de fortes contraintes pour que l'énergie dissipée soit suffisamment importante pour provoquer un échauffement notable (ce qui ne se produirait pas avec des matériaux peu résistants) et, d'autre part, que l'élévation de température correspondante diminue sensiblement la résistance, ce qui se produit plus facilement lorsqu'on est près du point de fusion. Cette instabilité peut engendrer des surfaces de glissement, par exemple lors du forgeage à chaud, et ces surfaces deviennent ultérieurement des zones de faiblesse, ou des amorces de rupture des pièces métalliques refroidies.

Le cas des argiles enfin paraît plus complexe car aucune modification de volume ou de température ne vient faire varier la résistance. Ici, le radoucissement, c'est-à-dire la diminution de résistance en fonction de la déformation après qu'un maximum ait été dépassé, correspond à la désorganisation d'une certaine texture de la matière, même si nous ne savons pas exactement quelles sont les liaisons qui ont faibli.

L'analogie de ces trois comportements est cependant très grande; le but de cette étude est de la mettre en évidence.

\section{RADOUCISSEMENT DES SABLES DENSES}

La figure 1 représente une courbe classique contraintedéplacement avec radoucissement pour un milieu pulvérulent compact au cours d'un essai triaxial et les variations de volume correspondantes.

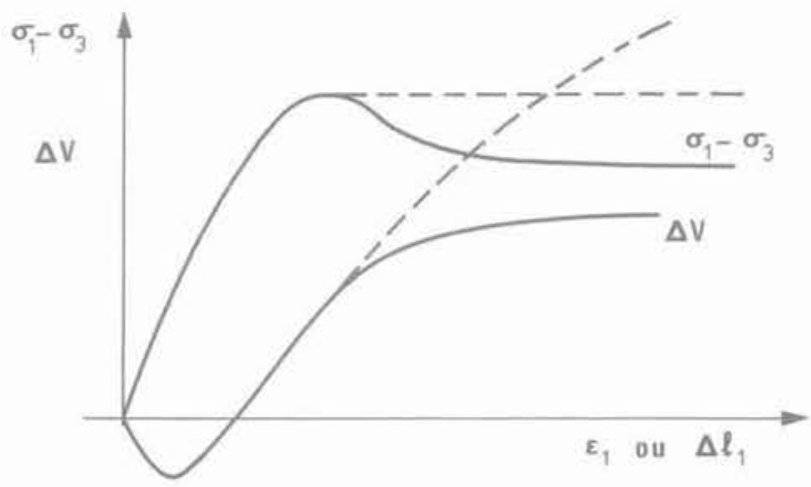

Fig. 1. - Comportement et variation de volume d'un sable dense dans un essai triaxial.

En champ uniforme, pour un sable très compact, il est généralement admis qu'une surface de glissement unique apparaît après le maximum et qu'il existe une liaison claire entre la dilatance et l'apparition des surfaces de glissement (BEEN et JEFFERIES, 1985). Cela ne veut pas dire qu'il ne se produit rien avant le maximum: la plupart des modèles théoriques indiquent que la localisation peut s'initialiser pendant l'écrouissage (MOLENKAMP, 1985). DUTHILLEUL (1983) a observé, sur des matériaux bidimensionnels, que la dilatance, d'abord très générale, se condense progressivement sous la forme d'une bande de cisaillement dont l'épaisseur diminue progressivement et qui finit par se matérialiser comme un plan de glissement au moment du passage du maximum. De toutes façons, les résultats expérimentaux ne permettent pas, pour l'instant, de choisir le bon modèle de comportement mais les expérimentateurs voient le plan de glissement apparaître immédiatement après le maximum de résistance.

Si l'on empêche la localisation de se développer, par exemple avec des échantillons courts et avec un « antifrettage des appuis, d'autres lignes de glissement apparaissent formant un double réseau et du même coup la dilatance continue de se produire, il n'y a plus de maximum de résistance jusqu'à des déformations de $40 \%$; après, on ne sait plus très bien ce qui se passe. On peut donc imaginer deux mécanismes de variation de volume. Le premier est une variation homogène dans l'éprouvette qui est d'abord une contraction, peut-être ensuite une dilatation. Le deuxième mécanisme est une dilatance localisée qui correspond à l'augmentation de volume de la bande de cisaillement, augmentation $\Delta V$ qui devient de plus en plus grande et tend vers une limite lorsque l'épaisseur de la bande de cisaillement diminue et se transforme en surface de glissement. 
Il est facile d'exprimer le travail de déformation associé au premier mécanisme dans l'essai triaxial. Il suffit d'intégrer dans les limites convenables :

$$
\begin{aligned}
\mathrm{dW} & =\sigma_{1} \mathrm{~d} \varepsilon_{1}+2 \sigma_{3} \mathrm{~d} \varepsilon_{3} \\
& =\left(\sigma_{1}-\sigma_{3}\right) \mathrm{d} \varepsilon_{1}+\sigma_{3} \mathrm{dV}
\end{aligned}
$$

à partir des données expérimentales de la figure 1 . Au début du chargement axial le travail croît linéairement avec la déformation qui est associée à une diminution du volume.

Le travail correspondant au deuxième mécanisme est plus difficile à évaluer car lorsqu'on approche de la rupture et qu'un plan de glissement se forme dans une direction $\beta$ la conduite de l'essai triaxial classique est telle que les composantes normale et tangentielle $n$ et $t$ de la contrainte sur la surface de glissement $\beta$ croissent simultanément (fig, 2).

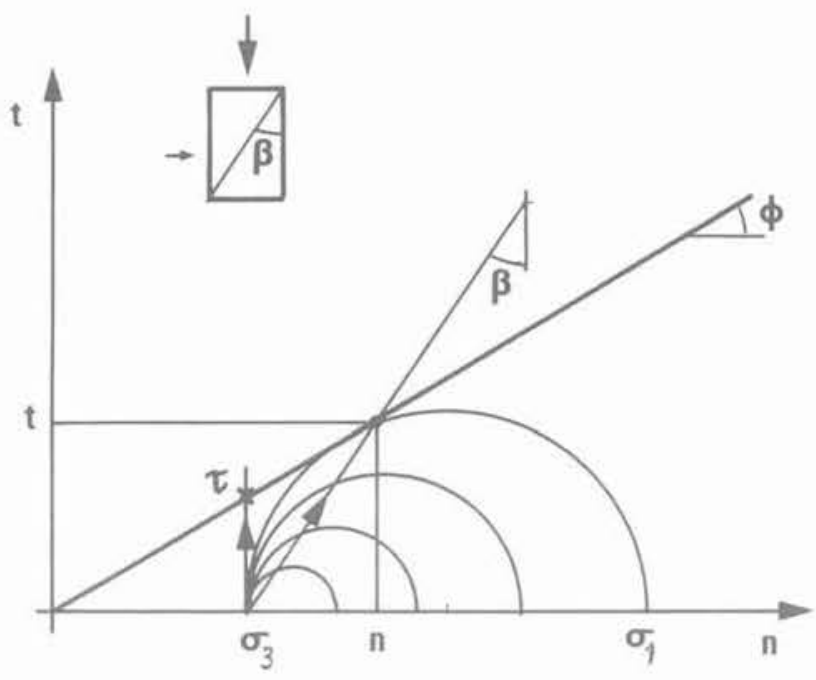

Fig. 2. - Trajet de chargement dans un essai triaxial.

De sorte que la tendance au désenchevêtrement des grains dans le plan de glissement est gênée par l'augmentation de la contrainte normale $n$ : on a peu de renseignements expérimentaux relatifs à ce mode de chargement. Il est donc beaucoup plus simple de se placer dans une situation de cisaillement analogue à celle de l'essai à la boîte de Casagrande (c'est-à-dire le trajet $\sigma_{3} \tau$ de la figure 2), avec une contrainte normale n constante sur la facette de glissement. C'est d'ailleurs cette situation qui a été examinée par les auteurs qui ont étudié l'instabilité thermique des métaux (POMEY, 1964 - BAQUÉ et FELDER, 1974). On peut envisager pour cela un essai de torsion sur tube dans des conditions de contraintes telles que la surface de glissement se produise dans un plan perpendiculaire à l'axe du tube : pour un métal répondant au critère de Tresca, ce serait une torsion simple; pour un sable avec un critère de Coulomb la contrainte axiale, normale à la section droite, serait légèrement plus faible que la contrainte intermédiaire $\sigma_{2}$. Enfin, la contrainte $\sigma_{2}$ sera choisie de façon que la déformation radiale $\varepsilon_{2}$ reste nulle pendant l'essai (déformation plane) : c'est cette configuration qui sera seule examinée dans la suite de cette étude. On peut donc représenter la distorsion par le schéma de la figure 3 avec $\gamma=\frac{\mathrm{x}}{\mathrm{H}}$ où $\mathrm{x}$ est le déplacement tangentiel dû à la rotation de la tête de l'éprouvette de hauteur $\mathrm{H} ; \mathrm{z}$ est le gonflement associé à la dilatance et il est supposé petit devant $\mathrm{H}$.

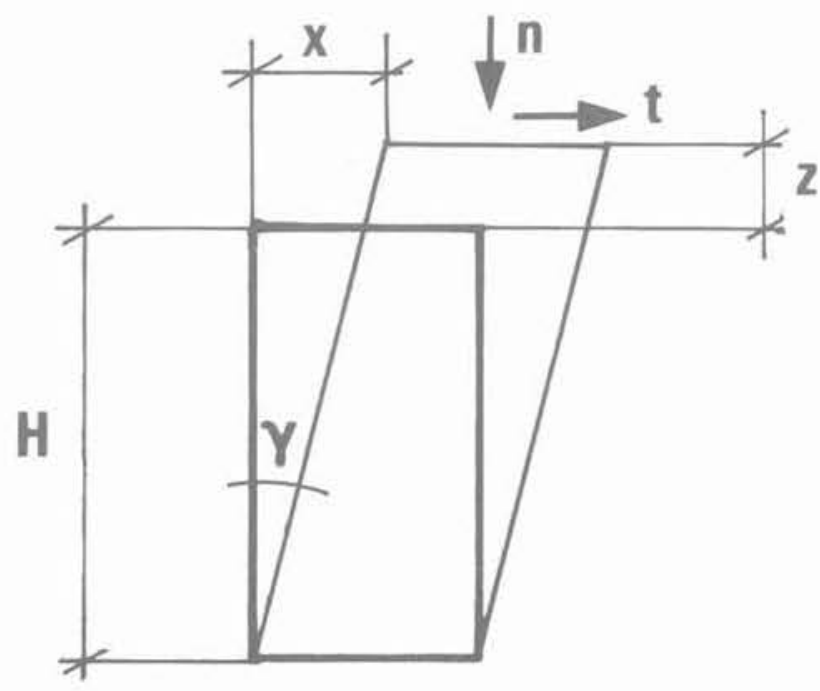

Fig. 3. - Essais de distorsion : notations.

On peut maintenant imaginer que le travail de déformation est composé de deux parties :

- lune correspond à l'écoulement plastique associé à la résistance au frottement résiduel;

- l'autre correspond à la variation de volume et son expression est particulièrement simple ici puisque c'est à tout instant $\int_{0}^{x} \mathrm{n} \cdot \mathrm{dz}$ avec $\mathrm{n}$ constant, ce qui n'est pas le cas dans l'essai triaxial classique.

a. Supposons d'abord que le cisaillement se produise sans variation de volume $(z=0)$. Pour un sable au voisinage de la densité critique, on peut exprimer la relation entre t et $\gamma$ de la figure 4 par une équation de la forme :

$$
\mathrm{t}=\mathrm{t}_{0}\left(1-\mathrm{e}^{-i \gamma}\right)
$$

avec par exemple $\lambda=20$, de façon que t soit de l'ordre de $90 \%$ de $t_{0}$ pour un glissement $\gamma=10 \%$ (fig. 4).

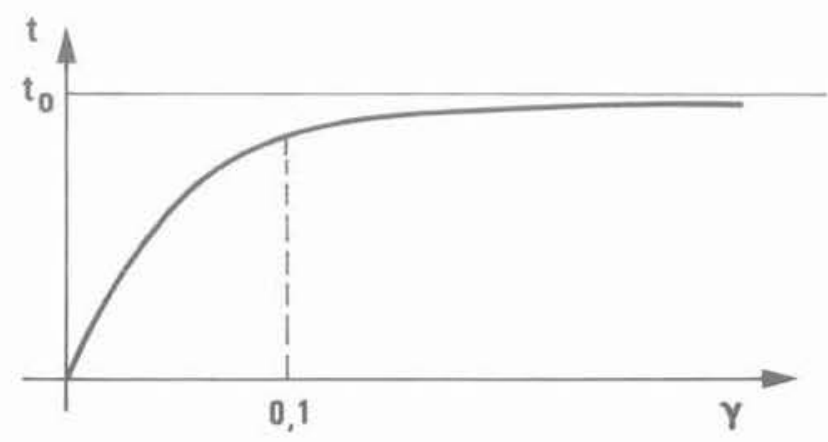

Fig. 4. - Cisaillement d'un sable à la densité critique. 
Le travail de la force $\mathrm{t}$ dans le déplacement $\mathrm{x}$ est :

$$
\begin{aligned}
W_{1} & =\int_{0}^{x} t_{0}\left(1-e^{-i \frac{x}{H}}\right) d x \\
& =t_{0}\left[x+\frac{H}{\lambda} e^{-i \frac{x}{H}}\right]_{0}^{x} \\
& =t_{0}\left[x+\frac{H}{\lambda} e^{-\lambda \frac{x}{H}}-\frac{H}{\lambda}\right]
\end{aligned}
$$

C'est une fonction de $\mathrm{H}$ dont la dérivée par rapport à $\mathrm{H}$ est :

$$
\begin{aligned}
\frac{d W_{1}}{d H} & =t_{0}\left(\frac{x}{H} e^{-\lambda \frac{x}{H}}+\frac{1}{\lambda} e^{-\lambda \frac{x}{H}}-\frac{1}{\lambda}\right) \\
& =t_{0}\left[e^{-\lambda \frac{x}{H}}\left(\frac{x}{H}+\frac{1}{\lambda}\right)-\frac{1}{\lambda}\right] \\
& =t_{0}\left[e^{-\lambda \gamma}\left(\gamma+\frac{1}{\lambda}\right)-\frac{1}{\lambda}\right]
\end{aligned}
$$

dont la dérivée par rapport à $\gamma$ :

$$
\begin{aligned}
\left(\frac{\mathrm{dW}}{\mathrm{dH}}\right)_{\gamma}^{\prime} & =-\lambda e^{-\lambda \gamma}\left(\gamma+\frac{1}{\lambda}\right)+e^{-\lambda \gamma} \\
& =-\lambda \gamma e^{-\lambda \gamma}
\end{aligned}
$$

est toujours négative dans $\gamma(0, \infty)$. La fonction $\mathrm{dW}_{1} / \mathrm{dH}$ est donc décroissante dans cet intervalle. Or $\mathrm{dW}_{1} / \mathrm{dH}(\gamma=0)=0$ donc $\mathrm{dW}_{1} / \mathrm{dH}<0$ dans $\gamma(0, \infty) . W_{1}$ est donc une fonction décroissante de $\mathrm{H}$ : l'énergie de déformation $W_{1}$ est d'autant plus petite que $\mathrm{H}$ est grand : cela signifie que le mode de défor- mation le plus facile est celui de la déformation homogène.

Un tel résultat est tout à fait évident dans le cas de l'élasticité linéaire $(t=a \gamma)$; le mécanisme de déformation (a) de la figure 5 qui comporte une amorce de localisation peut être engendré par un cisaillement complémentaire $\Delta \mathrm{t}$, mais on revient au mécanisme de la déformation homogène $(\mathrm{b})$ dès qu'on relâche $\Delta \mathrm{t}$. Si la relation entre t et $\gamma$ était linéaire mais non réversible, la déformation subsisterait après la suppression de $\Delta t$, mais elle serait effacée lorsque t augmentant dépasserait $t+\Delta t$ : c'est le schéma (c) de la figure 5 .

On obtient donc une situation analogue lorsque t est simplement une fonction croissante de $\gamma$.

b. Supposons maintenant que le matériau ne présente que de la dilatance. Le travail de déformation est $\mathrm{W}_{2}=\int_{0}^{x} \mathrm{n} \mathrm{dz}$ et avec $\mathrm{n}=$ Cte il vient $\mathrm{W}_{2}=\mathrm{n} \mathrm{z}$ $=n H \frac{Z}{H}=n H \varepsilon_{2}(\gamma)$. La fonction $\varepsilon_{2}(\gamma)$ est donnée approximativement par la figure 6 .

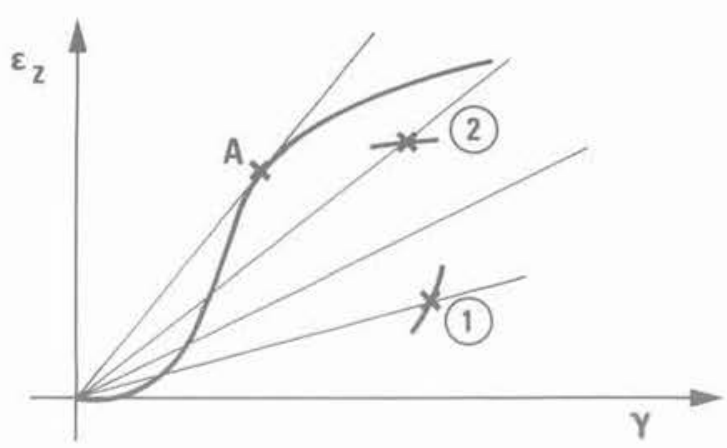

Fig. 6. - Comportement dilatant au cours du cisaillement.

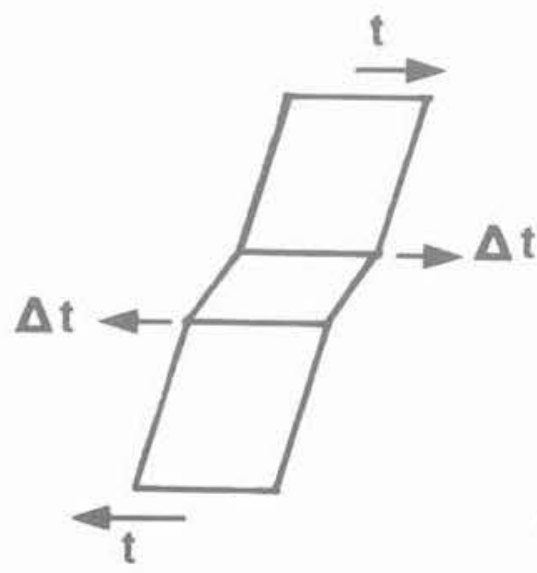

(a)

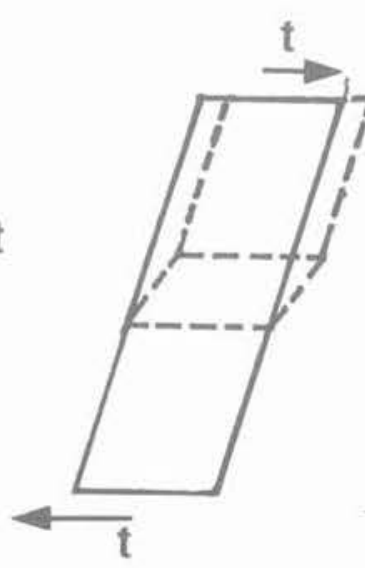

(b)

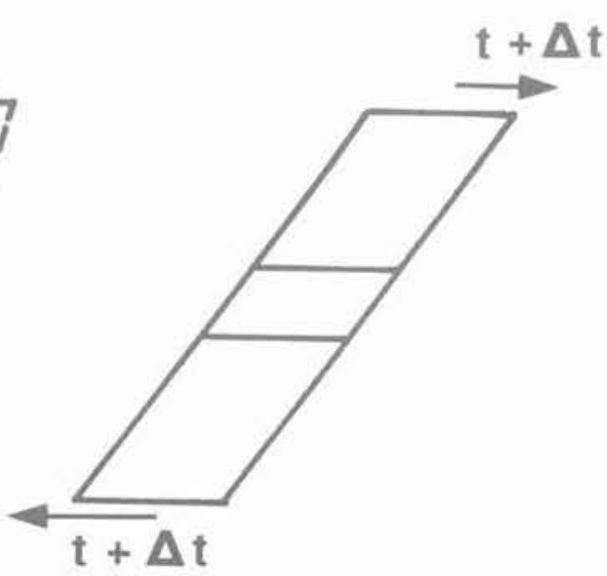

(c)

Fig. 5. - Localisation forcée. 
La dérivée :

$$
\frac{\mathrm{dW}_{2}}{\mathrm{dH}}=\mathrm{n}\left[-\frac{\mathrm{x}}{\mathrm{H}} \dot{\varepsilon}_{(y)}^{\prime}+\varepsilon_{(y-j)}\right]
$$

s'annule pour: $\varepsilon_{(y)}=\gamma \varepsilon_{(y)}^{\prime}$

équation différentielle à variables séparées :

$$
\frac{\mathrm{d} \varepsilon}{\varepsilon}=\frac{\mathrm{d} \gamma}{\gamma}
$$

dont la solution est $\varepsilon=\mathrm{C} \gamma$, c'est-à-dire le réseau de droites issues de l'origine de la figure 6 ; lorsqu'en un point de la courbe $\varepsilon_{\text {y }}$ on a $\varepsilon_{\text {' }}>\mathrm{C}$, on voit immédiatement que $\mathrm{dW}_{2} / \mathrm{dH}<0$, c'est-à-dire, comme nous l'avons vu plus haut, que le mécanisme de déformation le plus facile correspond à la déformation homogène (cas 1 de la figure 6). Par contre, si en un point de la courbe $\varepsilon_{(y)}$ on a $\varepsilon^{\prime}<\mathrm{C}$, on voit que $\mathrm{dW}_{2} / \mathrm{dH}>0$ (cas 2 de la figure 6). Le travail de déformation est d'autant plus faible que $\mathrm{H}$ est petit : la hauteur de la zone qui supporte le glissement $\gamma$ tend à diminuer et il y a localisation de la déformation sur une surface de glissement. Pour une courbe dont la forme est celle de la figure 6 , c'est à partir du point $A$ qu'apparaît la localisation.

c. Le cas général est maintenant facile à traiter. L'énergie de déformation est $\mathrm{W}=\mathrm{W}_{1}+\mathrm{W}_{2}$. Il faut que :

$$
\begin{aligned}
\frac{d W}{d H}=\frac{d W_{1}}{d H}+\frac{d W_{2}}{d H}= & t_{0}\left[e^{-i \gamma}\left(\gamma+\frac{1}{\lambda}\right)-\frac{1}{\lambda}\right] \\
& +n\left(\varepsilon_{\langle y\rangle}-\gamma \varepsilon_{(y)}^{\prime}\right)>0
\end{aligned}
$$

pour avoir une condition de localisation, ou encore :

$$
\varepsilon_{(y)}-\gamma \dot{\varepsilon}_{(y)}^{\prime}>\frac{t_{0}}{n}\left[\frac{1}{\lambda}-e^{-\lambda \gamma}\left(\frac{1}{\lambda}+\gamma\right)\right]
$$

$$
\operatorname{Or} U_{(y)}=\frac{t_{0}}{n}\left[\frac{1}{\lambda}-e^{-\lambda \gamma}\left(\frac{1}{\lambda}+\gamma\right)\right] \text { est toujours }
$$

croissant puisque sa dérivée $\lambda e^{-\lambda \gamma}\left(\frac{1}{\lambda}+\gamma\right)-e^{-i \gamma}$

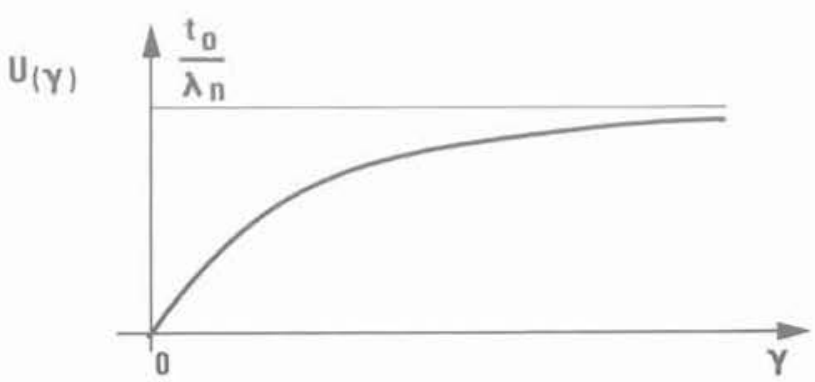

Fig. 7. - Fonction U(Y). $=\lambda \gamma e^{-i \gamma}$ est toujours positive dans $\gamma(0, \infty)$. Comme $\mathrm{U}_{(\gamma=0)}=0, \mathrm{U}_{(y)}$ est toujours positif dans $\gamma(0, \infty)$ et sa plus grande valeur $t_{0} / \lambda \mathrm{n}$ est obtenue pour $\gamma \rightarrow \infty$ (figure 7).

Par conséquent, si :

$$
\varepsilon_{(y)}-\gamma \dot{\varepsilon}_{(y)}^{\prime}>\frac{t_{0}}{\lambda \mathrm{n}}
$$

la condition (1) est vérifiée. On peut écrire cette dernière relation :

$$
\frac{\varepsilon_{(y)}-\frac{t_{0}}{\lambda n}}{\gamma}>\varepsilon_{(y)}^{\prime}
$$

ou encore

$$
\frac{\mathrm{d} \gamma}{\gamma}>\frac{\mathrm{d} \varepsilon}{\varepsilon_{(\gamma)}-\frac{\mathrm{t}_{0}}{\lambda \mathrm{n}}}
$$

L'équation différentielle correspondante s'intègre en :

$$
\begin{array}{ll}
\ln \left(\varepsilon-\frac{t_{0}}{\lambda \mathrm{n}}\right) & =\ln (\gamma)+\ln (C)=\ln (C \gamma) \\
\text { ou : } \quad & \varepsilon=C \gamma+\frac{t_{0}}{\lambda n}
\end{array}
$$

dont la représentation est le faisceau de droite issu du point $\left(0, \frac{t_{0}}{\lambda \mathrm{n}}\right)$ de la figure 8 . Ce point est d'autant plus proche de lorigine 0 que $n$ est grand.

Un raisonnement analogue à celui du paragraphe précédent montre que c'est à partir du point $\left(A^{\prime}\right)$ de la courbe $\varepsilon_{(-,)}$que le localisation de la déformation se produit.

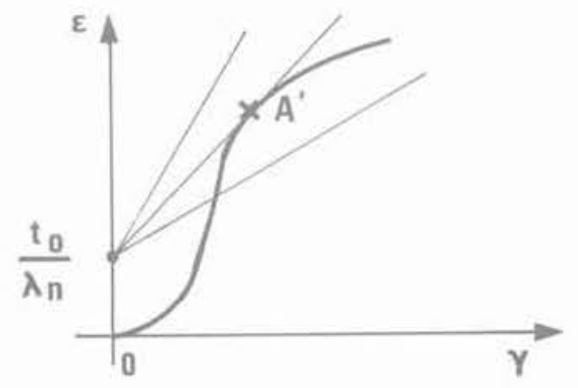

Fig. 8. - Comportement d'un sable dilatant au cours du cisaillement.

\section{RADOUCISSEMENT DES MATÉRIAUX THERMOSENSIBLES}

Les matériaux thermosensibles qui sont envisagés ici sont ceux dont la résistance au cisaillement t est une fonction de la température T. En général, lorsqu'il n'y a 
pas de changement de phase, la résistance diminue avec la température et ce phénomène est d'autant plus marqué qu'on est près de la température de fusion (figure 9).

Dans le cas de la déformation plastique des métaux à haute température, l'observation a montré que ce phénomène est susceptible de provoquer l'apparition d'une ou de plusieurs bandes de glissement plus chaudes que le milieu environnant, ou même, de faire apparaître des surfaces de glissement à partir du mécanisme catastrophique suivant: la déformation plastique augmente localement la température, ce qui diminue localement la résistance. Dans un champ de contrainte hétérogène une surface de glissement peut donc se développer et progresser de la même manière que pour les sables denses (P. HABIB, 1984). Dans un champ de contrainte homogène, on a une situation d'instabilité. Cet effet est pratiquement insensible pour les matériaux peu résistants comme l'argile, la pâte à modeler ou les matières plastiques, car le travail dissipé plastiquement y est insuffisant pour élever notablement la température, sauf dans le cas des chargements cycliques (M.P. LUONG, 1982) où l'on peut avoir un effet cumulatif. Dans les roches profondes au cours d'un séisme, c'est-à-dire lors de la localisation du glissement sur une faille, il se produit probablement un radoucissement d'origine thermique, dans certains cas jusqu'à la fusion de la roche puisqu'on a retrouvé des produits de vitrification.

L'interprétation du phénomène de radoucissement thermique est simple. Le travail dW engendré par la déformation plastique de cisaillement se transforme en la quantité de chaleur $\frac{\mathrm{dW}}{\mathrm{J}}$ qui engendre une augmentation de température $\mathrm{dT}$ :

$$
\begin{aligned}
d W & =t_{(T)} d x \\
& =J d T \cdot H \cdot \text { l.e.p.c, }
\end{aligned}
$$

où $/$ et e sont la largeur et l'épaisseur de zone cisaillée : on peut leur donner, par exemple, des valeurs unitaires; $\rho$ est la masse volumique et c la chaleur spécifique. Posons $\mu=J /$ e $\rho$ c pour simplifier l'écriture.

Si $\mathrm{t}=\mathrm{t}_{0}-\mathrm{m} \mathrm{T}$ est considéré comme une bonne approximation du radoucissement thermique entre $A$ et $B$, sur la figure 9 on $a$ :

$$
\begin{gathered}
\left(\mathrm{t}_{0}-\mathrm{m} \mathrm{T}\right) \mathrm{dx}=\mu \mathrm{HdT} \\
\frac{\mathrm{dx}}{\mu \mathrm{H}}=-\frac{1}{\mathrm{~m}} \frac{\mathrm{d}\left(\mathrm{t}_{0}-\mathrm{m} \mathrm{T}\right)}{\mathrm{t}_{0}-\mathrm{mt}} \\
\mathrm{x}=-\frac{\mu \mathrm{H}}{\mathrm{m}} \ln \left(\frac{1}{\mathrm{C}}\left(\mathrm{t}_{0}-\mathrm{mT}\right)\right) \\
\mathrm{t}_{0}-\mathrm{m} \mathrm{T}=\mathrm{C} e^{-\frac{\mathrm{mx}}{\mu \mathrm{H}}} \text { et } \mathrm{C}=\mathrm{t}_{0} \\
\operatorname{car} \mathrm{T}_{(\mathrm{x})}=0 \text { pour } \mathrm{x}=0 \\
\mathrm{~T}(\mathrm{x})=\frac{\mathrm{t}_{0}}{\mathrm{~m}}\left(1-e^{-\frac{\mathrm{mx}}{\mu \mathrm{H}}}\right) .
\end{gathered}
$$

Le travail de déformation étant $\mathrm{W}=\mu \mathrm{HT}$, on $\mathrm{a}$ :

$$
\mathrm{W}=\frac{\mu \mathrm{Ht}_{0}}{\mathrm{~m}}\left(1-\mathrm{e}^{-\frac{\mathrm{mx}}{\mu \mathrm{H}}}\right) .
$$

C'est à nouveau une fonction de $\mathrm{H}$ dont la dérivée est :

$$
\frac{\mathrm{m}}{\mu \mathrm{t}_{0}} \frac{\mathrm{dW}}{\mathrm{dH}}=1-\mathrm{e}^{-\frac{\mathrm{mx}}{\mu \mathrm{H}}}\left(1+\frac{\mathrm{mx}}{\mu} \frac{1}{\mathrm{H}}\right) .
$$

Sa représentation est indiquée sur la figure 10 .
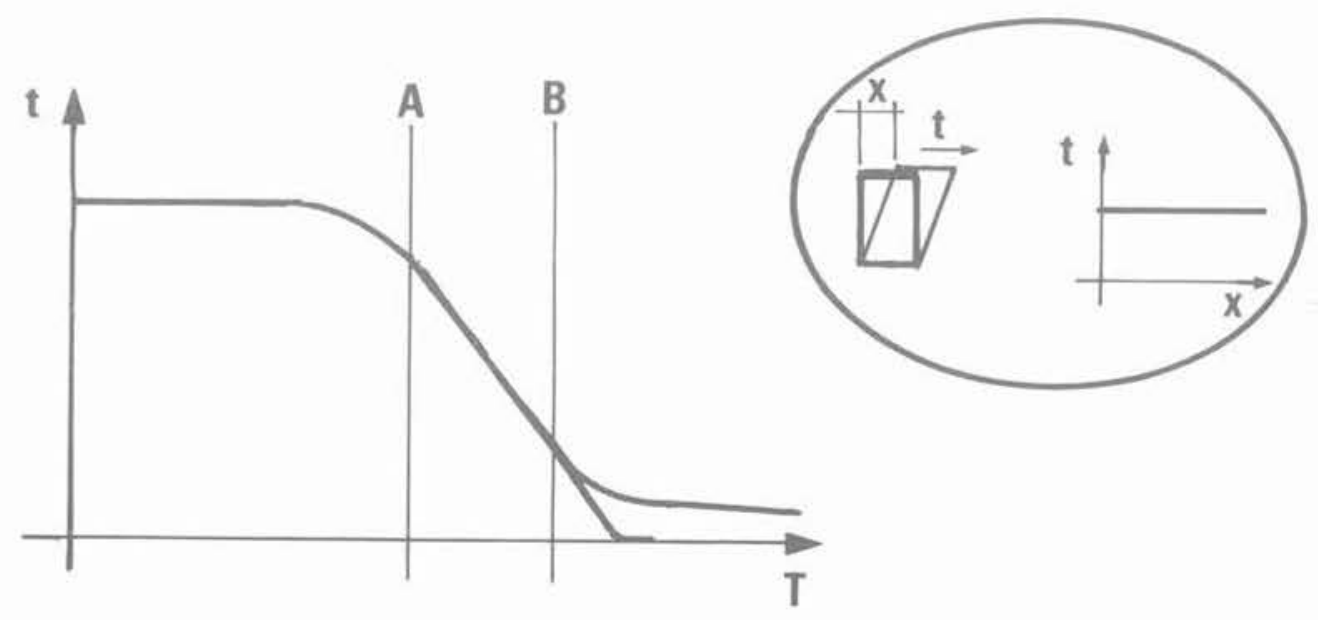

Fig. 9. - Variation de la résistance au cisaillement $t$ en fonction de la température $T$. 


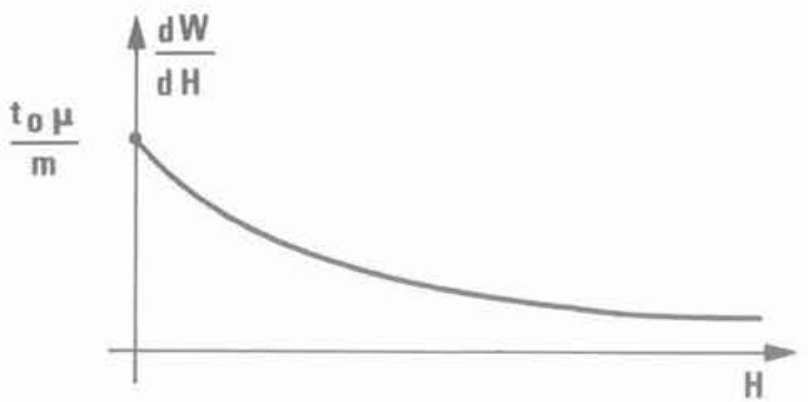

Fig. 10. $-\frac{d W}{d H}$ en fonction de $H$.

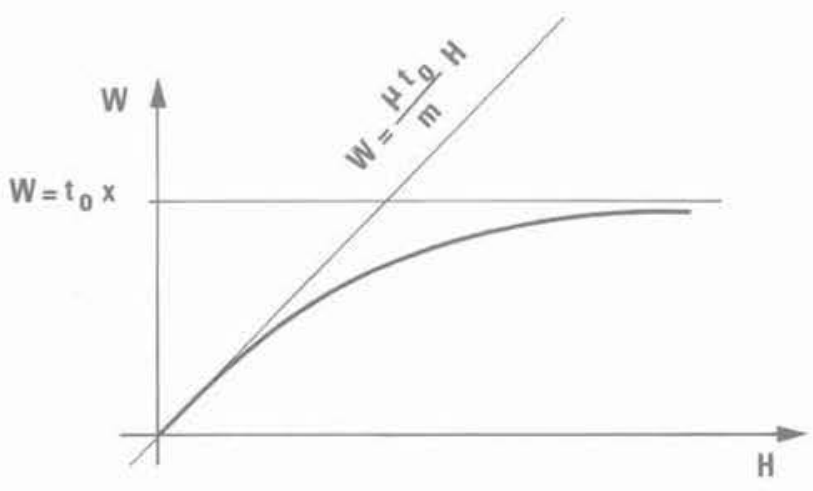

Fig. 11. - W en fonction de $\mathrm{H}$.
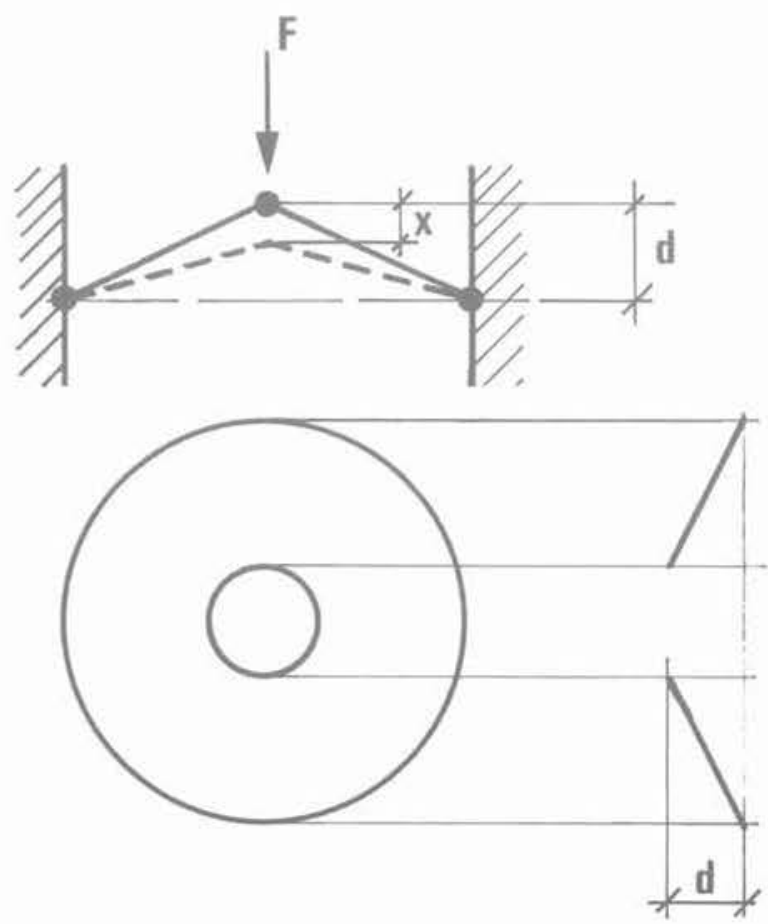

Elle est toujours positive dans $\mathrm{H}(0, \infty)$ : W est donc une fonction croissante de $\mathrm{H}$ et le mécanisme de déformation le plus facile correspond à une localisation de la déformation. Sur la figure 11, le fait que la courbe $\mathrm{W}(\mathrm{H})$ passe par l'origine correspond à ce qu'on a négligé la diffusion thermique ce qui permet d'accepter une bande de cisaillement d'épaisseur nulle et n'a évidemment aucune signification physique.

On remarquera que $t$ décroissant avec $T$ entraîne $t$ décroissant avec $\gamma$.

\section{COMMENTAIRE}

Nous venons de rencontrer plusieurs cas où la décroissance de la résistance au cisaillement en fonction du glissement $\gamma$ entraîne la croissance du travail de déformation $W$ avec l'épaisseur $H$ de la zone cisaillée. Dans ces exemples, il en résultait que le mécanisme de déformation le plus aisé correspondait à une épaisseur décroissante, à la limite à une surface de glissement où la distorsion est infiniment grande. Arrêtons-nous un instant sur la signification physique de ce phénomène.

Lorsqu'il se produit, il y a passage progressif et successif d'un mécanisme à un autre, sans discontinuité, comme il s'en produit lorsqu'on passe du régime d'écoulement hydraulique laminaire à l'écoulement turbulent et réciproquement : ici il n'y a pas d'équilibre métastable. En particulier il n'y a pas de retour

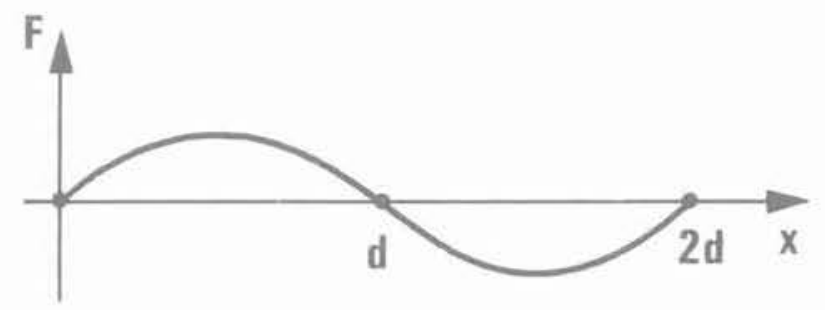

Fig. 12. - Comportement d'une rondelle Belleville. 
en arrière comme en élasticité, comme cela est illustré sur la figure 5 où l'on passait d'une configuration à une autre en fonction des forces appliquées. Ceci pourrait peut-être se produire si l'on avait une courbe t $\gamma$ radoucissante réversible. On pourrait alors prévoir le retour vers la position déformée du corps présentant l'énergie potentielle la plus faible. Mais, est-ce vraiment possible?

\section{Essayons donc de construire un modèle.}

Pour obtenir un comportement radoucissant, il suffit de faire appel aux grandes déformations des structures. On peut citer, par exemple, le dispositif composé de deux bielles élastiques avec troís articulations, dont deux fixes, ou la coque appelée «rondelle Belleville». La figure 12 indique la courbe effort-déplacement de telles structures, comme on peut l'obtenir au cours d'un essai à déplacement contrôlé. Lorsque le déplacement $x$ est égal à l'épaisseur initiale d, la structure est aplatie et la force est nulle; après un déplacement de $2 \mathrm{~d}$, la force devenue négative est à nouveau nulle, l'énergie restituée est égale, au frottement près, à l'énergie emmagasinée et le système est revenu à l'état initial (à une rotation de $\pi$ près).

La première partie de la courbe de comportement est donc élastique réversible non linéaire radoucissante et a priori rien n'empêche de construire un dispositif avec une butée pour empêcher le phénomène de cloquage.

Voyons maintenant ce qui se passe si l'on superpose deux structures de ce type (figure 13.a). La partie croissante de la courbe effort-déplacement se déduit de la précédente par une affinité dans la direction de l'axe des déplacements. Le déplacement est doublé jusqu'au maximum mais comme les deux structures ne sont pas strictement identiques, la plus faible s'effondre la première pendant que l'autre se redresse. Si la
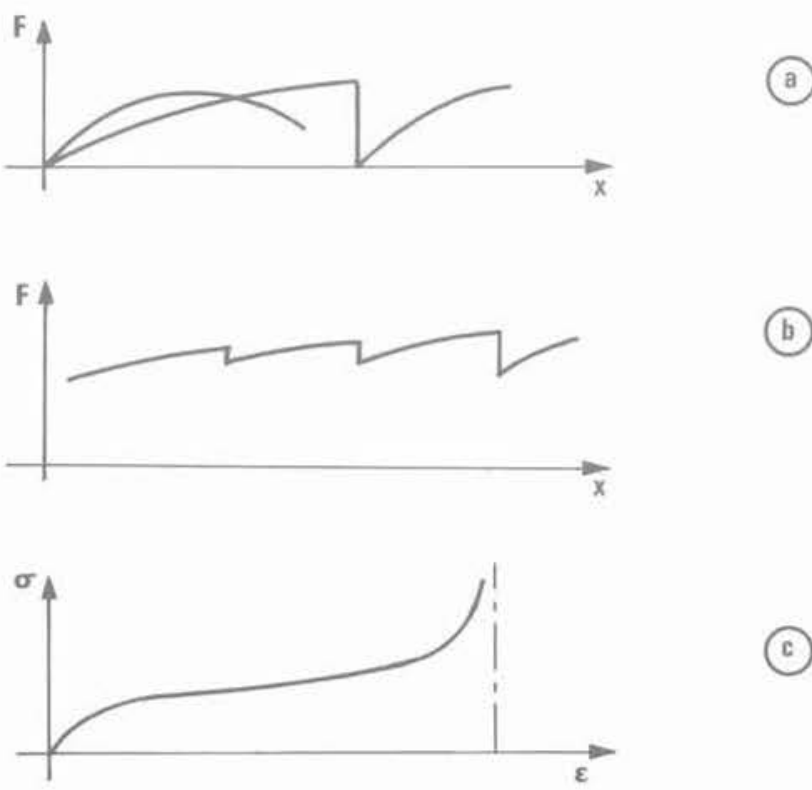

Fig. 13. - Comportement d'un empilement. courbe de la figure 12 est symétrique, l'énergie emmagasinée par la structure qui s'aplatit est strictement égale à celle qui est restituée par la structure qui se détend et la force disparaît sans aucun déplacement. L'ensemble du système est revenu sous charge nulle et si l'on augmente à nouveau le déplacement, on reproduit le début de la courbe de la figure 12 .

Si l'on superpose $\mathrm{n}$ structures du même type, on obtiendra un ressort à très faible complaisance, le premier effondrement d'une des n structures se traduit par une diminution de $1 / n^{\text {eme }}$ de $F$, etc. (figure 13.b), puis se termine par la courbe de la figure 13. a pour les deux dernières structures: ces comportements sont certes intéressants, mais ne sont nullement radoucissants.

Supposons maintenant qu'il existe un matériau dont le comportement $\mathrm{f}(\sigma, \varepsilon)$ soit élastique non linéaire radoucissant, c'est-à-dire réversible, il suffit de découper, par la pensée, une éprouvette en n morceaux en série pour retrouver un empilement de structures analogue au précédent et reproduire le phénomène de la figure 13, dans la mesure où la matière n'est jamais parfaitement homogène (ou les morceaux jamais tout à fait égaux). On démontre ainsi que le comportement radoucissant réversible n'existe pas, non pas pour des raisons thermodynamiques mais pour des raisons de stabilité.

Terminons ce commentaire par une dernière remarque relative au comportement des corps réels où la déformation interne correspond aux grands déplacements d'une structure (débobinage des macromolécules d'un élastomère, flambage des cloisons d'un matériau spongieux, cloquage d'un empilage de rondelles, etc.). Dans la réalité, le phénomène élémentaire se termine par un blocage (ou par l'inversion des formes dans le cas des rondelles de Belleville) et le matériau se raidit progressivement. On aboutit alors aux courbes sigmoïdes bien connues pour le bois en compression, le caoutchouc en traction, etc., avec une asymptote verticale pour la force, où se situe la rupture par destruction (figure 13.c).

En conclusion, le radoucissement ne peut correspondre qu'à des déformations irréversibles donc à une certaine forme de plasticité.

\section{RADOUCISSEMENT DES MATÉRIAUX ARGILEUX}

Le phénomène du radoucissement est d'autant plus marqué pour les argiles que la pression de consolidation est grande, par exemple supérieure à 0,3 ou à $0,4 \mathrm{MPa}$. Mais, pour les argiles saturées, contrairement aux cas précédents, il n'y a ni variation de volume ni ramollissement d'origine thermique. La variation de résistance au cisaillement est indiquée sur la figure 14 . On peut l'approximer par morceaux par les droites :

$$
\begin{array}{ll}
\mathrm{t}=\mathrm{m} \gamma & \text { dans la partie écrouissable, } \\
\mathrm{t}=\mathrm{Cte} & \text { au maximum et au palier, } \\
\mathrm{t}=\mathrm{t}_{0}-\mathrm{m} \gamma & \text { dans la partie radoucissante. }
\end{array}
$$




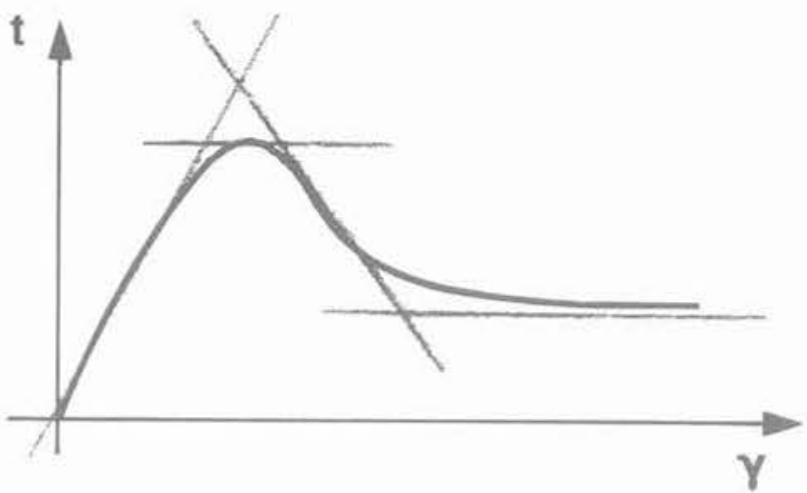

Fig. 14. - Comportement d'une argile.

Il est plus simple de traiter chaque cas séparément.

a.

$$
t=m \frac{x}{H}:
$$

Le travail $\mathrm{T}=\int_{0}^{x} \mathrm{t} d x=\frac{\mathrm{mx}^{2}}{2 \mathrm{H}}$ est une fonction hyperbolique décroissante de $\mathrm{H}$ : comme nous l'avons vu antérieurement la déformation se généralise à toute P'éprouvette.

b.

$$
\mathrm{t}=\text { Cte : }
$$

$\mathrm{W}=\int_{0}^{x} \mathrm{cdx}=\mathrm{cx}$ est une fonction indépendante de $\mathrm{H}$ : la déformation en cours se poursuit.

c.

$$
\mathrm{t}=\mathrm{t}_{0}-\frac{\mathrm{mx}}{\mathrm{H}}:
$$

$\mathrm{W}=\int_{0}^{\mathrm{x}}\left(\mathrm{t}_{0}-\frac{\mathrm{mx}}{\mathrm{H}}\right) \mathrm{dx}=\mathrm{t}_{0} \mathrm{x}-\frac{\mathrm{mx^{2 }}}{2 \mathrm{H}}$ est une fonction croissante de $\mathrm{H}$ dans $\mathrm{H}(0, \infty)$; il y a localisation de la déformation. On ne peut évidemment avoir $\mathrm{W}<0$ donc $\mathrm{H}<\mathrm{m} x / 2 \mathrm{t}_{0}$ : il est bien clair qu'il s'agit là d'un artefact qui vient de la loi de comportement adoptée telle qu'à partir de $\mathrm{H}=\mathrm{m} \times / \mathrm{t}_{0}$ le cisaillement devient négatif, ce qui n'a pas de sens physique. Un tel incident disparaitrait avec une 'approximation moins grossière et, par exemple, avec une courbe admettant une asymptote parallèle à l'axe des abscisses, mais cela ne changerait pas les résultats qualitatifs de la présente analyse.

\section{MATÉRIAUX ÉLASTOPLASTIQUES PARFAITS}

Pour déterminer le comportement élastoplastique parfait représenté sur la figure 15.a, il est courant d'effectuer des essais de compression simple sur éprouvettes cylindriques. Au cours d'une telle expérience, la hauteur de l'éprouvette diminue et la section droite augmente. Le diagramme Force-Déplacement présente alors un écrouissage apparent (figure 15.b). Si on avait effectué un essai de traction simple on aurait eu un effet inverse et la section droite de l'éprouvette diminuant le diagramme Force-Déplacement aurait présenté un radoucissement (figure 15.c). C'est d'ailleurs à partir des essais 15 . b et 15 .c et moyennant les corrections de sections appropriées, qu'on déduit la courbe de comportement 15.a. On peut qualifier cet écrouissage et ce radoucissement de géométrique.

On voit immédiatement dans l'essai de compression que la déformation est homogène et qu'elle reste cylindrique si les conditions d'appui sont sans frottement. Par contre, dans la traction, la déformation est instable et on sait que l'essai se termine par une rupture en striction dont la parenté avec le phénomène de formation d'une surface de glissement est évidente : l'emplacement de la striction est aléatoire et sa localisation ne s'explique que par l'hétérogénéité de la matière, la rupture s'amorçant dans la section la plus faible de l'éprouvette.

\section{DISCUSSION}

Il n'est pas possible d'attribuer l'homogénéité de la déformation en compression ou l'instabilité de la traction et la localisation de la striction au comportement physique de la matière. On pourrait d'ailleurs concevoir un essai de traction instable (c'est-à-dire se terminant par une striction) avec une loi de comportement présentant un peu d'écrouissage. On pourrait aussi empêcher la rupture localisée et stabiliser la déformation d'allongement en utilisant des matériaux qui peuvent durcir dans certaines conditions par exemple par effet de viscosité (superplasticité) ou par certains effets thermiques (filage du verre).

Ces considérations autour de l'essai de traction laissent planer un doute sur le fait que l'apparition d'une surface de glissement soit liée, dans les matériaux que

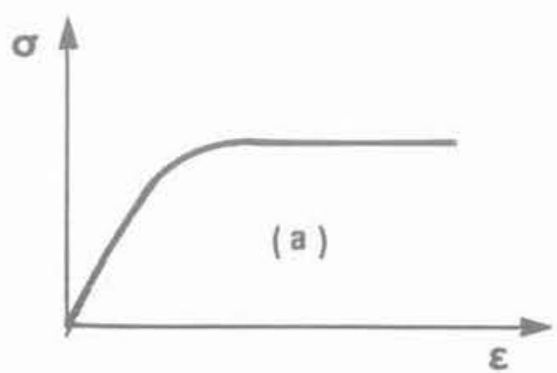

$\boldsymbol{\varepsilon}$

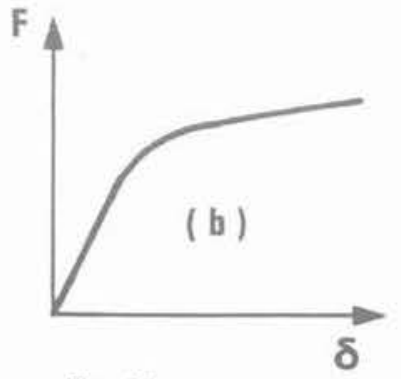

Fig. 15:

a. Comportement élastoplastique parfait.

b. Essai réel de compression.

c. Essai réel de traction.

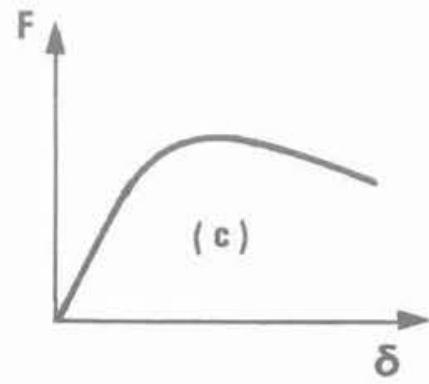


nous avons étudiés précédemment, à certains phénomènes physiques comme la dilatance des sables denses, les effets thermodynamiques des roches (ou des métaux thermosensibles) ou la destruction d'une texture dans le cas des argiles. En fait l'apparition d'une surface de glissement en cisaillement provient simplement du radoucissement et en se replaçant dans le protocole expérimental de la figure 3 , on s'aperçoit que tous les raisonnements qui ont été faits ici reviennent simplement à évaluer le travail dissipé au cours d'une déformation de cisaillement par :

$W=\int_{0}^{x} t(x) d x$ qui est une fonction croissante de $x ;$

$t(x)$ est connu par une expérience de glissement permettant de relier $t$ à $\gamma$ par une expression $t\left(\frac{x}{H}\right)$.

Dans ces conditions :

$$
W=\int_{0}^{x} t\left(\frac{x}{H}\right) d x
$$

est une fonction de $\mathrm{H}$ et pour en connaitre le sens de variation, il suffit de dériver sous le signe somme :

$$
\begin{aligned}
\frac{d W}{d H} & =\int_{0}^{x} t^{\prime}{ }_{\gamma} \gamma_{x}^{\prime} d x=-\int_{0}^{x} t^{\prime} \frac{x}{H^{2}} d x \\
& =-\int_{0}^{x} t^{\prime}, \frac{x}{H} \frac{d x}{H} \\
& =-\int_{0}^{x} t^{\prime} \gamma d \gamma
\end{aligned}
$$

qui s'intègre immédiatement par partie :

$$
\frac{d W}{d H}=\int_{0}^{x} t(\gamma) d \gamma-\gamma t(\gamma)
$$

dont l'interprétation géométrique est simple : si t $(\gamma)$ est décroissant (fig. 16.a) le rectangle $\gamma t(\gamma)$ est plus petit que laire sous la courbe $t(\gamma), \frac{\mathrm{dW}}{\mathrm{dH}}$ est alors positif, W est une fonction croissante de $\mathrm{H}$, il y a localisation.

Si $t(\gamma)$ est croissant le rectangle $\gamma t(\gamma)$ est plus grand que l'aire sous la courbe $t(\gamma), \frac{\mathrm{dW}}{\mathrm{dH}}$ est négatif, $\mathrm{W}$ est une fonction décroissante de $\mathrm{H}$ et la déformation est généralisée.

Dans l'expression du travail donnée plus haut, rien n'empêche d'évaluer $W$ entre des bornes autres que o et $\mathrm{x}$, par exemple entre $\mathrm{x}_{1}$ et $\mathrm{x}_{2}$ ou mème $\mathrm{x}$ et $\mathrm{x}+\Delta \mathrm{x}$ :

$$
\Delta W=\int_{x}^{x+\Delta x} t\left(\frac{x}{H}\right) d x=t\left(\frac{x}{H}\right) \Delta x
$$

qui est encore une fonction de $\mathrm{H}$ et dont la dérivée est :

$$
\frac{d \Delta W}{d H}=-t^{\prime}(\gamma) \frac{x}{H^{2}} \Delta x
$$

qui est du signe de $-t^{\prime}(\gamma)$ : lorsque la fonction $t(\gamma)$ est croissante $\frac{\mathrm{d} \Delta \mathrm{W}}{\mathrm{dH}}$ est une fonction décroissante et la déformation est homogène; lorsque $t(\gamma)$ est décroissante $\frac{\mathrm{d} \Delta \mathrm{W}}{\mathrm{dH}}$ est une fonction croissante et il $\mathrm{y}$ a localisation. Ce résultat est évidemment à rapprocher du fait que $\frac{\mathrm{dW}}{\mathrm{dx}}$ est la force de cisaillement.

\section{CONCLUSION}

Les différents matériaux qui ont été successivement examinés ont tous la propriété d'avoir une résistance qui diminue avec la déformation à partir d'un certain seuil. Cet effet a été clairement identifié comme une irréversibilité plastique et c'est particulièrement évident pour les matériaux thermosensibles. Dans le schéma expérimental de la déformation plane de cisaillement, le radoucissement entraîne l'apparition d'une surface de glissement localisée; l'énergie de déformation est minimale pour la ruine de léprouvette mais la matière n'est pas complètement détruite pour autant puisqu'il subsiste deux parties monolithiques qui ont conservé leurs propriétés mécaniques initiales mais qui sont séparées l'une de l'autre par une surface où la résis. tance de la matière est amoindrie.
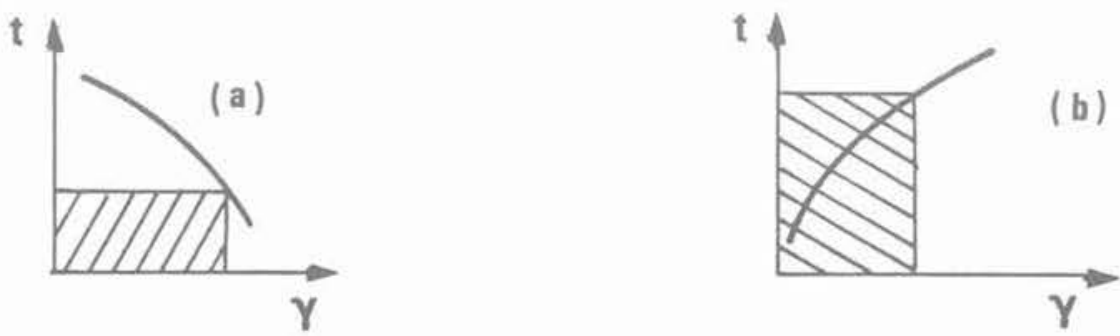

Fig. 16. - Condition de localisation. 


\section{BIBLIOGRAPHIE}

1. BAQUÉ P. et FELDER E. (1974), Instabilité d'écoulements thermoviscoplastiques, Annals of the CIRP, vol. 23/1, 1974, pp. 79-80.

2. BEEN K. and JEFFERIES M.G. (1985), A state parameter for sands. Géotechnique, vol. XXXV, number 2, June 1985, pp. 99-125.

3. DUTHILLEUL B. (1983), Rupture progressive : simulation physique et numérique (Thèse DocteurIngénieur, INPG, Grenoble).

4. EVANS B. and WONG T.F. (1983), Shear localisation in rocks induced by tectonic deformation. William Prager Symposium, Mechanics of Geomaterials, Rocks, Concretes, Soils. Northwestern Uni- versity, Evanston (Illinois), 11 septembre 1983, pp. 191-219.

5. HABIB P. (1984), Les surfaces de glissement en Mécanique des Sols, Revue Française de Géotechnique, $n^{\circ} 27$ (2e trimestre 1984).

6. LUONG M.P. (1982), Détection par thermographie infrarouge du seuil caractéristique d'un sable cisaillé en vibrations, C.R. Acad. Sci., Paris, t. 295, série II, pp. 87-89 (20 sept. 1982).

7. MOLENKAMP F. (1985), Comparison of frictional material models with respect to shear band initiation, Géotechnique, vol. XXXV, number 2, June 1985, pp. 127-143.

8. POMEY J. (1964), Sur un phénomène de plasticité adiabatique, Note aux C.R. Acad. Sci. (Paris). t. 258, 27 avril 1964, Groupe 2, pp. 4204-4205. 